\title{
Identificação de aflatoxinas no leite e produtos lácteos: Revisão de literatura
}

\author{
Identification of aflatoxins in millk and dairy products: Literature review \\ Identificación de aflatoxinas en la leche y los productos lácteos: Revisión de la literatura
}

Recebido: 26/06/2021 | Revisado: 04/07/2021 | Aceito: 06/07/2021 | Publicado: 17/07/2021

\author{
Laura Soares Magalhães \\ ORCID: https://orcid.org/0000-0003-2413-6319 \\ Universidade Federal dos Vales do Jequitinhonha e Mucuri, Brasil \\ E-mail:laura.magalhaes@ufvjm.edu.br \\ Marília Cristina Sola \\ ORCID: https://orcid.org/0000-0002-2125-4064 \\ Universidade Federal dos Vales do Jequitinhonha e Mucuri, Brasil \\ E-mail: marilia.sola@ufvjm.edu.br
}

\begin{abstract}
Resumo
As aflatoxinas são metabólitos tóxicos de fungos do gênero Aspergillus que podem ser comumente encontradas em produtos de origem animal e vegetal. O objetivo deste trabalho foi investigar a presença de aflatoxinas no leite e seus derivados a partir da consulta de trabalhos publicados no Brasil através das bases de dados Scientific Eletronic Library Online, Pubmed e Google Scholar. Para isso, foram escolhidos dezesseis trabalhos publicados entre os anos de 1989 e 2018 onde treze avaliaram o leite em diferentes formas de tratamento e três avaliaram queijos. Dos treze estudos acerca da detecção de aflatoxinas no leite, cinco apresentaram uma concentração acima do limite estabelecido pela legislação brasileira para aflatoxinas em leite, sendo 0,010 a $0,645 \mu \mathrm{g} / \mathrm{L}, 0,075$ a $1,280 \mu \mathrm{g} / \mathrm{L}, 1,68 \mu \mathrm{g} / \mathrm{L}, 0,04$ a 1,05 $\mu \mathrm{g} / \mathrm{L}$ e 0,04 a $4,64 \mu \mathrm{g} / \mathrm{L}$. Dos três estudos que avaliaram queijos, todos verificaram a presença de aflatoxinas, mas apenas um estudo identificou concentração do metabólito acima do limite máximo tolerado, resultando num valor entre 2,7 e $6,6 \mu \mathrm{g} / \mathrm{kg}$. A metodologia utilizada para detecção da toxina fúngica variou entre as técnicas CLAE, CCD, CLUE e ELISA. A presença das aflatoxinas em produtos lácteos demonstra a importância do monitoramento desses produtos, bem como uma maior atenção aos alimentos destinados aos animais de produção, sendo esta, fonte de contaminação indireta para o homem, podendo gerar impacto à saúde por seu consumo de forma crônica. Assim sendo, torna-se evidente a necessidade de um monitoramento constante das aflatoxinas nos produtos de origem animal, tendo em vista a ocorrência natural e elevada toxicidade das aflatoxinas para a saúde humana e animal.
\end{abstract}

Palavras-chave: Aspergillus spp.; Derivados lácteos; Micotoxinas; Resíduos químicos.

\begin{abstract}
Aflatoxins are toxic metabolites of fungi of the Aspergillus genus that can be commonly found in animal and vegetable products. The objective of this study was to investigate the presence of aflatoxins in milk and its derivatives based on the consultation of works published in Brazil through the Scientific Electronic Library Online, Pubmed and Google Scholar databases. For this, sixteen papers published between 1989 and 2018 were chosen, where thirteen evaluated milk in different forms of treatment and three evaluated cheese. Of the thirteen studies on the detection of aflatoxins in milk, five showed a concentration above the limit established by the Brazilian legislation for aflatoxins in milk, being 0.010 to $0.645 \mu \mathrm{g} / \mathrm{L}, 0.075$ to $1.280 \mu \mathrm{g} / \mathrm{L}, 1.68 \mu \mathrm{g} / \mathrm{L}, 0.04$ to $1.05 \mu \mathrm{g} / \mathrm{L}$ and 0.04 to $4.64 \mu \mathrm{g} / \mathrm{L}$. Of the three studies that evaluated cheese, all verified the presence of aflatoxins, but only one study identified a metabolite concentration above the maximum tolerated limit, resulting in a value between 2.7 and $6.6 \mu \mathrm{g} / \mathrm{kg}$. The methodology used to detect the fungal toxin varied between the HPLC, CCD, CLUE and ELISA techniques. The presence of aflatoxins in dairy products demonstrates the importance of monitoring these products, as well as greater attention to food intended for farm animals, which is a source of indirect contamination for humans, which can impact health by chronic consumption. Therefore, the need for constant monitoring of aflatoxins in products of animal origin becomes evident, considering the natural occurrence and high toxicity of aflatoxins for human and animal health.
\end{abstract}

Keywords: Aspergillus spp.; Dairy derivatives; Mycotoxins; Chemical residues.

\section{Resumen}

Las aflatoxinas son metabolitos tóxicos de hongos del género Aspergillus que se pueden encontrar comúnmente en productos animales y vegetales. El objetivo de este estudio fue investigar la presencia de aflatoxinas en la leche y sus derivados a partir de la consulta de trabajos publicados en Brasil a través de las bases de datos Scientific Electronic Library Online, Pubmed y Google Scholar. Para ello, se eligieron dieciséis artículos publicados entre 1989 y 2018 , donde trece evaluaron la leche en diferentes formas de tratamiento y tres evaluaron el queso. De los trece estudios 
sobre la detección de aflatoxinas en la leche, cinco mostraron una concentración por encima del límite establecido por la legislación brasileña para las aflatoxinas en la leche, siendo de 0,010 a 0,645 $\mu \mathrm{g} / \mathrm{L}, 0,075$ a 1,280 $\mu \mathrm{g} / \mathrm{L}, 1,68 \mu \mathrm{g} / \mathrm{L}$, 00,04 a $1,05 \mu \mathrm{g} / \mathrm{L}$, y 0,04 a 4,64 $\mu \mathrm{g} / \mathrm{L}$. De los tres estudios que evaluaron el queso, todos verificaron la presencia de aflatoxinas, pero solo un estudio identificó una concentración de metabolitos por encima del límite máximo tolerado, lo que arrojó un valor entre 2,7 y $6,6 \mu \mathrm{g} / \mathrm{kg}$. La metodología utilizada para detectar la toxina fúngica varió entre las técnicas de HPLC, CCD, CLUE y ELISA. La presencia de aflatoxinas en los productos lácteos demuestra la importancia de monitorear estos productos, así como una mayor atención a los alimentos destinados a los animales de granja, que es una fuente de contaminación indirecta para el hombre, que puede afectar la salud por el consumo crónico. Por lo tanto, se hace evidente la necesidad de un monitoreo constante de las aflatoxinas en los productos de origen animal, considerando la ocurrencia natural y la alta toxicidad de las aflatoxinas para la salud humana y animal.

Palabras clave: Aspergillus spp.; Derivados lácteos; Micotoxinas; Residuos químicos.

\section{Introdução}

As micotoxinas são metabólitos secundários produzidos por algumas espécies de fungos, sendo considerados contaminantes naturais de difícil controle nos alimentos. Apesar de serem descritas com maior frequência em produtos de origem vegetal, os alimentos de origem animal também podem ser comprometidos.

De acordo com Bochio et al. (2017), a maioria dos produtos animais podem ser contaminados a partir da ingestão das micotoxinas presentes nas rações, gerando assim um resíduo tecidual. A contaminação do leite, por exemplo, ocorre após a metabolização da toxina através de enzimas hepáticas. No fígado, a aflatoxina B1, ingerida pelo animal através de rações contaminadas, ao alcançar o fígado, sofre uma biotransformação, resultando em aflatoxina M1, que é excretada junto ao produto lácteo (Hussein \& Brasell, 2001).

Atualmente no Brasil, há grande oferta e demanda de produtos de origem animal. O país é um dos maiores produtores de leite do mundo, mantendo-se em quarto lugar no ranking mundial. Em 2020, a produção leiteira industrializada atingiu 25,4 bilhões de litros, tendo Minas Gerais como estado destaque, produzindo 24,6\% do total (IBGE, 2020). Devido a essa magnitude de produção e consumo, fez-se necessário a realização de estudos acerca da presença de micotoxinas no leite, sendo concretizados em todo o mundo.

Além do leite, as micotoxinas podem ser encontradas em outros produtos de origem animal, como derivados lácteos, produtos cárneos e ovos. Desse modo, o estudo a respeito da ocorrência de micotoxinas nesses alimentos, bem como no leite e seus derivados, apresenta grande importância para a sociedade por apresentar risco à saúde humana e animal, acarretando perdas econômicas, devido à desvalorização e descarte dos alimentos contaminados.

\section{Metodologia}

A metodologia utilizada neste estudo foi a revisão bibliográfica, realizada entre os meses de fevereiro e abril de 2021 a fim de identificar a presença de aflatoxinas no leite e produtos lácteos.

As principais bases de dados online consultadas foram Scientific Eletronic Library Online (https://scielo.org/), Pubmed (https://pubmed.ncbi.nlm.nih.gov/), Ministério da Saúde (http://bvsms.saude.gov.br/) e Google Scholar (https://scholar.google.com/) por meio da busca pelos termos "aflatoxin bovine milk", "mycotoxins present in products of animal origin", "presença de aflatoxinas em produtos lácteos", "aspergillus flavus", "timely conditions for the production of mycotoxins", "effects of mycotoxins in animals", "fungi characterization", "zearalenone in dairy products", "ochratoxins in dairy cows" e "fumonisin in milk".

Foram analisados 16 trabalhos entre os anos de 1989 e 2018 que realizaram a quantificação de micotoxinas no leite e derivados lácteos no Brasil. Estudos adicionais, entre os anos de 1980 e 2021 foram utilizados para enriquecer a introdução e discussão deste material. 


\section{Análise Estatística}

Empregou-se a estatística descritiva com a organização e apresentação dos dados por meio de tabela (distribuição de frequências e porcentagens para variáveis qualitativas) gerada no software Excel® versão 2013.

\section{Resultados e Discussão}

No Quadro 1 encontram-se os estudos que quantificaram micotoxinas em amostras de leite e derivados lácteos, sendo especificado o tipo de micotoxina, alimento avaliado, número de amostras avaliadas, \% de detecção, quantidade do analito detectado ( $\mu \mathrm{g} / \mathrm{L}$ ), limite de referência nacional, método de detecção, local do estudo e referência.

A partir dos resultados dispostos abaixo, foi possível notar que os estudos avaliados ocorreram entre os anos de 1989 e 2018 , sendo a detecção das micotoxinas realizada majoritariamente pelo método de cromatografia, com destaque à cromatografia líquida de alta eficiência.

Quadro 1. Presença de aflatoxinas em leite e queijos no Brasil entre os anos de 1989 e 2018.

\begin{tabular}{|c|c|c|c|c|c|c|c|c|}
\hline Micotoxina & $\begin{array}{c}\text { Alimento } \\
\text { Inspecionado }\end{array}$ & $\begin{array}{c}N^{\circ} \text { amostras } \\
\text { avaliadas }\end{array}$ & $\begin{array}{l}\% \text { de detecção } \\
\text { de micotoxinas }\end{array}$ & $\begin{array}{c}\text { Quantidade } \\
\text { detectada } \\
(\mu \mathrm{g} / \mathrm{L})\end{array}$ & $\begin{array}{c}\text { Limite de } \\
\text { referência } \\
\text { (BRASIL, 2021) }\end{array}$ & $\begin{array}{c}\text { Metodologia } \\
\text { utilizada }\end{array}$ & Local & Referências \\
\hline \multicolumn{9}{|c|}{ Leite in natura } \\
\hline $\begin{array}{l}\text { Aflatoxina M1 } \\
\text { (AFM1) }\end{array}$ & $\begin{array}{l}\text { Leite fluido in } \\
\text { natura }\end{array}$ & 65 & $40 \%$ & $\begin{array}{c}0,009 \text { a } 0,069 \\
\mu \mathrm{g} / \mathrm{L}\end{array}$ & $0,5 \mu \mathrm{g} / \mathrm{kg}$ & CLAE & $\begin{array}{l}\text { Pirassununga, } \\
\text { São Paulo }\end{array}$ & Jager, 2013 \\
\hline $\begin{array}{l}\text { Aflatoxina M1 } \\
\text { (AFM1) }\end{array}$ & Leite cru & 30 & $36,7 \%$ & $\begin{array}{c}0,010 \text { a } 0,645 \\
\mu \mathrm{g} / \mathrm{L}^{*}\end{array}$ & $0,5 \mu \mathrm{g} / \mathrm{kg}$ & CLAE & São Paulo & $\begin{array}{c}\text { Oliveira } \text { et al., } \\
2010\end{array}$ \\
\hline $\begin{array}{l}\text { Aflatoxina M1 } \\
\text { (AFM1) }\end{array}$ & Leite cru & 40 & $87,5 \%$ & $\begin{array}{c}0,013 \text { a } 0,018 \\
\mu \mathrm{g} / \mathrm{L}\end{array}$ & $0,5 \mu \mathrm{g} / \mathrm{kg}$ & ELISA & Castro, PR & Venâncio, 2017 \\
\hline $\begin{array}{l}\text { Aflatoxina M1 } \\
\text { (AFM1) }\end{array}$ & Leite in natura & 20 & $100 \%$ & $\begin{array}{c}0,075 \text { a } 1,280 \\
\mu \mathrm{g} / \mathrm{L}^{*}\end{array}$ & $0,5 \mu \mathrm{g} / \mathrm{kg}$ & $\begin{array}{l}\text { QuEChERS e } \\
\text { CCD }\end{array}$ & Concórdia, SC & $\begin{array}{l}\text { Gonçalves et al., } \\
2016 .\end{array}$ \\
\hline $\begin{array}{l}\text { Aflatoxina M1 } \\
\text { (AFM1) }\end{array}$ & Leite in natura & 100 & $1 \%$ & $1,68 \mu \mathrm{g} / \mathrm{L}^{*}$ & $0,5 \mu \mathrm{g} / \mathrm{kg}$ & CLAE & $\begin{array}{l}\text { Vale Médio do } \\
\text { Paraíba, SP }\end{array}$ & Sabino et al., 1989 \\
\hline \multicolumn{9}{|c|}{ Leite pasteurizado } \\
\hline $\begin{array}{l}\text { Aflatoxina M1 } \\
\text { (AFM1) }\end{array}$ & $\begin{array}{l}\text { Leite pasteurizado } \\
\text { tipo C }\end{array}$ & 40 & $42,5 \%$ & $\begin{array}{c}0,02 \text { a } 0,14 \\
\mu \mathrm{g} / \mathrm{kg}\end{array}$ & $0,5 \mu \mathrm{g} / \mathrm{kg}$ & CCD & Curitiba, PR. & Baggio, 2006. \\
\hline $\begin{array}{l}\text { Aflatoxina M1 } \\
\text { (AFM1) }\end{array}$ & Leite pasteurizado & 107 & $74 \%$ & $\begin{array}{c}0,02 \text { a } 0,26 \\
\mu \mathrm{g} / \mathrm{L}\end{array}$ & $0,5 \mu \mathrm{g} / \mathrm{kg}$ & CCD & $\begin{array}{l}\text { São Paulo e } \\
\text { Marília, SP }\end{array}$ & $\begin{array}{c}\text { Shundo e Sabino, } \\
2006 .\end{array}$ \\
\hline
\end{tabular}


Research, Society and Development, v. 10, n. 8, e50510817586, 2021

(CC BY 4.0) | ISSN 2525-3409 | DOI: http://dx.doi.org/10.33448/rsd-v10i8.17586

\begin{tabular}{|c|c|c|c|c|c|c|c|c|}
\hline $\begin{array}{l}\text { Aflatoxina M1 } \\
\text { (AFM1) }\end{array}$ & Leite pasteurizado & 43 & $39,5 \%$ & $\begin{array}{c}0,04 \text { a } 4,64 \\
\mu \mathrm{g} / \mathrm{L}^{*}\end{array}$ & $0,5 \mu \mathrm{g} / \mathrm{kg}$ & CLAE & São Paulo & $\begin{array}{c}\text { Gonçalez et al., } \\
2005\end{array}$ \\
\hline \multicolumn{9}{|c|}{ Leite UHT } \\
\hline $\begin{array}{l}\text { Aflatoxina M1 } \\
\text { (AFM1) }\end{array}$ & Leite UHT & 31 & $22,6 \% \%$ & $\begin{array}{c}0,0 \text { a } 0,183 \\
\mu \mathrm{g} / \mathrm{L}\end{array}$ & $0,5 \mu \mathrm{g} / \mathrm{kg}$ & CLAE/DFL & $\begin{array}{c}\text { Rio de Janeiro, } \\
\text { RJ }\end{array}$ & Castro et al., 2013 \\
\hline $\begin{array}{l}\text { Aflatoxina M2 } \\
\text { (AFM2) }\end{array}$ & Leite UHT & 16 & $18 \%$ & $0,009 \mu \mathrm{g} / \mathrm{L}$ & - & CLUE-EM/EM & Rio de Janeiro & Sartori et al., 2015 \\
\hline $\begin{array}{l}\text { Aflatoxina M1 } \\
\text { (AFM1) }\end{array}$ & $\begin{array}{c}\text { Leite UHT } \\
\text { desnatado }\end{array}$ & 11 & $63,6 \%$ & $\begin{array}{c}0,04 \text { a } 1,05 \\
\mu \mathrm{g} / \mathrm{L}^{*}\end{array}$ & $0,5 \mu \mathrm{g} / \mathrm{kg}$ & CLAE-FD & $\begin{array}{c}\text { Rio Grande, } \\
\text { Rio Grande do } \\
\text { Sul }\end{array}$ & Gonçalves, $2018^{\mathrm{b}}$ \\
\hline \multicolumn{9}{|c|}{ Leite em pó } \\
\hline $\begin{array}{l}\text { Aflatoxina M1 } \\
\text { (AFM1) }\end{array}$ & $\begin{array}{l}\text { Leite em pó } \\
\text { integral }\end{array}$ & 72 & $24 \%$ & $\begin{array}{c}0,08 \text { a } 1,19 \mu \mathrm{g} / \\
\mathrm{kg}\end{array}$ & $5 \mu \mathrm{g} / \mathrm{kg}$ & CLUE-EM/EM & Rio de Janeiro & Sartori et al., 2015 \\
\hline $\begin{array}{l}\text { Aflatoxina M1 } \\
\text { (AFM1) }\end{array}$ & Leite em pó & 7 & $100 \%$ & $\begin{array}{c}0,33 \text { a } 0,81 \\
\mu \mathrm{g} / \mathrm{L}\end{array}$ & $5 \mu \mathrm{g} / \mathrm{kg}$ & ELISA & Londrina, PR & Santos, 2015 \\
\hline \multicolumn{9}{|c|}{ Queijos } \\
\hline $\begin{array}{l}\text { Aflatoxina M1 } \\
\text { (AFM1) }\end{array}$ & Queijos coloniais & 15 & $100 \%$ & $\begin{array}{c}0,06 \text { a } 0,56 \\
\mu \mathrm{g} / \mathrm{kg}\end{array}$ & $2,5 \mu \mathrm{g} / \mathrm{kg}$ & ELISA & $\begin{array}{c}\text { Vale do } \\
\text { Taquari, RS }\end{array}$ & Saraiva, 2017 \\
\hline $\begin{array}{l}\text { Aflatoxina M1 } \\
\text { (AFM1) }\end{array}$ & Queijo & 10 & $30 \%$ & $\begin{array}{c}0,091 \text { a } 0,30 \\
\mu \mathrm{g} / \mathrm{kg}\end{array}$ & $2,5 \mu \mathrm{g} / \mathrm{kg}$ & CLAE & $\begin{array}{c}\text { Pirassununga } \\
\text { São Paulo }\end{array}$ & Jager, 2013 \\
\hline $\begin{array}{l}\text { Aflatoxina M1 } \\
\text { (AFM1) }\end{array}$ & Queijo Parmesão & 88 & $46,4 \%$ & 2,7 a $6,6 \mu \mathrm{g} / \mathrm{kg}^{*}$ & $2,5 \mu \mathrm{g} / \mathrm{kg}$ & $\begin{array}{c}\text { Cromatografia } \\
\text { por } \\
\text { imunoafinidade }\end{array}$ & Minas Gerais & Prado et al., 2008 \\
\hline
\end{tabular}

CCD: Cromatografia em camada delgada; CLAE: Cromatografia líquida de alta eficiência; CLUE-EM/EM: cromatografia líquida de ultra eficiência acopladas à espectrometria de massas sequencial; CLAE/DFL: Cromatografia Líquida de Alta Eficiência com Deteç̧ão por 2 Fluorescência; CLAE-FD: Cromatografia líquida de alta eficiência com detecção de fluorescência; QuEChERS: Quick, Easy, Cheap, Effective, Rugged, Safe. *Concentração $(\mu \mathrm{g} / \mathrm{kg})$ acima do estabelecido pela legislação vigente (Brasil, 2021).

Fonte: Autores (2021). 
A concentração dos metabólitos variou entre $0,009 \mu \mathrm{g} / \mathrm{L}$ e 1,68 $\mu \mathrm{g} / \mathrm{L}$ para leite in natura; $0,02-4,64 \mu \mathrm{g} / \mathrm{L}$ em amostras de leite pasteurizado; 0-1,05 $\mu \mathrm{g} / \mathrm{L}$ leite UHT; 0,08-1,19 $\mu \mathrm{g} / \mathrm{kg}$ em amostras de leite em pó e de 0,06-6,6 $\mu \mathrm{g} / \mathrm{kg}$ em queijos.

Dos treze estudos que avaliaram diferentes tipos de leite, como in natura, pasteurizado, em pó e UHT, cinco ultrapassaram a concentração máxima permitida de aflatoxina M1 conforme estabelecido pela legislação brasileira $(0,5 \mu \mathrm{g} / \mathrm{L})$ (BRASIL, 2021), sendo os estudos realizados por Sabino et al. (1989), Gonçalez et al. (2005), Oliveira et al. (2010), Gonçalves et al. (2016) e Gonçalves (2018b), indicando percentuais de deteç̧ão entre $0,01 \mu \mathrm{g} / \mathrm{L}$ e 4,65 $\mu \mathrm{g} / \mathrm{L}$.

Três estudos também avaliaram a presença de aflatoxina M1 em queijos, sendo que somente Prado et al. (2008) constataram valores superiores ao estabelecido pela legislação $(2,5 \mu \mathrm{g} / \mathrm{kg}$ ) (Brasil, 2021), obtendo valores entre 2,7 $\mu \mathrm{g} / \mathrm{kg}$ e 6,6 $\mu \mathrm{g} / \mathrm{kg}$, resultando em $46,4 \%$ das amostras contaminadas.

\section{Discussão}

As micotoxinas são produtos tóxicos produzidos por diversos fungos e que eventualmente podem ser encontradas em produtos de origem animal, como carnes, ovos, leite e derivados, devido a biotransformação ocorrida após a ingestão pelos animais.

Uma vez no organismo, a toxina fúngica passa por uma metabolização hepática podendo se depositar nos músculos, o que acarreta sua presença em produtos cárneos, assim como ocorre a biotransformação sob micotoxinas primárias (AFB1) possibilitando a excreção via leite (AFM1) (Tittlemier et al., 2020). Isso demonstra a importância do monitoramento destes produtos, bem como o manejo correto dos alimentos dos animais de produção para que sejam livres de quaisquer contaminantes que ofereçam risco à saúde.

Com base nos resultados avaliados neste estudo, foi possível observar que, mesmo após o leite ter sido beneficiado termicamente, seja pela pasteurização ou esterilização, esses métodos não foram eficientes na eliminação destes metabólitos, demonstrando a dificuldade em controlá-los uma vez que já estejam presentes nos alimentos (Zheng et al., 2013).

Há diferenças significativas na regulamentação de micotoxinas em nível nacional e internacional, onde União Europeia se destaca por apresentar legislação mais rigorosa, apresentando um nível máximo de $0,05 \mu \mathrm{g} / \mathrm{L}$ para leite in natura e $0,03 \mu \mathrm{g} / \mathrm{L}$ para leite em pó (EC, 2016), seguida dos Estados Unidos com limites máximos de 0,5 $\mu \mathrm{g} / \mathrm{L}$ para AFM1 em leite integral, semi-desnatado e desnatado (FDA, 2013). Já no Brasil, a Instrução Normativa nº8 da ANVISA estabelece o limite máximo de AFM1 em 0,5 $\mu \mathrm{g} / \mathrm{L}$ para leite fluido; $5,0 \mu \mathrm{g} / \mathrm{L}$ para leite em pó e 2,5 $\mu \mathrm{g} / \mathrm{kg}$ em queijos, sendo estes parâmetros também seguidos para países do MERCOSUL (Brasil, 2021).

Quanto ao monitoramento destes metabólitos em produtos lácteos no país, o Ministério da Agricultura, Pecuária e Abastecimento (MAPA) por meio do Plano Nacional de Controle de Resíduos e Contaminantes em produtos de origem animal (PNCRC) verifica anualmente, a partir de uma amostragem baseada em análise de risco, a presença de resíduos de substâncias químicas potencialmente nocivas à saúde humana, como resíduos de produtos de uso veterinário, agrotóxicos e outros contaminantes químicos. Quanto as aflatoxinas no leite, foram avaliadas 910 amostras, entre os anos de 2010 e 2019, apresentando resultados expressivos de 98,34\% de conformidade (limite de referência - 0,50 $\mu \mathrm{g} / \mathrm{L}$ ) (Brasil, 1999), contrastando com os resultados observados neste estudo.

Neste mesmo sentido, relatos de Gonçalves et al. (2016) reforçam a importância das pesquisas relacionadas a aflatoxinas em produtos lácteos, ao afirmarem que $80 \%$ das amostras avaliadas apresentaram-se acima do limite estabelecido pela legislação brasileira. Essa porcentagem elevada demonstra a necessidade de se avaliar a presença de micotoxinas nos produtos de origem animal, uma vez que são conhecidos os impactos que causam à saúde humana. 
Assim como a aflatoxina M1, a aflatoxina M2 realiza o papel de um importante metabólito de excreção ativa em animais. No país, não há limites máximos tolerados para aflatoxina M2 em alimentos, o que se torna um fator preocupante, tendo em vista que estudos feitos no Brasil, como o de Sartori e colaboradores (2015), demonstraram a presença deste metabólito em amostras de leite UHT. Da mesma maneira, Lamiaa e colaboradores (2019), através da Alexandria University no Egito, detectaram uma incidência de $20 \%$ de contaminação de aflatoxina M2 no queijo Mish e 13,3\% no queijo Ras, comprovando que se trata de um metabólito presente não só no território brasileiro e que também oferece riscos à saúde humana.

Apesar da escassez de informações quanto à detecção de micotoxinas como a fumonisina, zearalenona e ocratoxina no leite e derivados quando comparado às aflatoxinas e da ausência de limites máximos tolerados na legislação brasileira, esses metabólitos podem impactar negativamente a saúde humana e animal, sendo necessário seu monitoramento, visto a presença em substratos utilizados para a elaboração de rações voltadas a alimentação de animais de produção (Huang et al., 2014; IQBAL et al., 2014).

Considerando os impactos que as aflatoxinas podem gerar na saúde humana e animal, o Codex Alimentarius (FAO, 2007) recomenda a adoção de práticas de prevenção destes metabólitos em grãos como estratégia de controle na produção de alimentos para consumo animal e consequentemente minimizar a presença nos produtos de consumo humano. Além das estratégias para controle e armazenamento adequado dos produtos, tem sido adotado a incorporação de substâncias para inativação das micotoxinas em cereais como a amônia, hidróxido de sódio, formaldeído e metilaminas, entretanto, estes podem impactar no valor nutricional dos alimentos (Baptista et al. (2002; Dias, 2018).

A aplicação de vapores de amônio e a irradiação dos alimentos também pode ser adotados como forma preventiva, entretanto, a adição de leveduras como Saccharomyces cerevisiae na alimentação de animais, tem sido eficaz na redução da transferência de aflatoxinas M1 para o leite (Gonçalves, 2018a). Deste modo, isso demonstra a existência de uma ótima solução para diminuir a veiculação deste metabólito para humanos, tendo em vista que a toxina se mostra resistente aos tratamentos térmicos empregados pela indústria.

\section{Considerações Finais}

A partir da avaliação de estudos publicados no Brasil, entre os anos de 1989 e 2018, as aflatoxinas M1 estiveram presentes em amostras de leite e derivados, sendo detectadas principalmente por métodos cromatográficos, indicando a necessidade de monitoramento constante destas substâncias nos produtos de origem animal, diante da ocorrência natural e elevada toxicidade das aflatoxinas.

\section{Referências}

Brasil. (1999). Ministério da Agricultura, Pecuária e Abastecimento. Instrução Normativa n² 42 de 20 de dezembro de 1999 . Dispõe sobre o Plano Nacional do Controle de Resíduos em Produtos de Origem Animal -PNCR e os Programas de Controle de Resíduos em Carne - PCRC, Mel - PCRM, Leite - PCRL e Pescado - PCRP. Diário Oficial da União.

Brasil.(2021). Ministério da Saúde/ Agência Nacional de Vigilância Sanitária/ Diretoria Colegiada. Instrução Normativa n. 88 de 2021. Dispõe sobre os limites máximos de tolerados de contaminantes em alimentos. Diário Oficial da União.

Baggio, E. C. R. (2006). Determinação de aflatoxina $\mathrm{m} 1$ em leite pasteurizado pelos métodos de CCD e CLAE utilizando coluna de imunoafinidade. Programa de Pós-Graduação em Tecnologia de Alimentos. Setor de Tecnologia. Universidade Federal do Paraná.

Baptista, A. S., Horii, J., Calori-Domingues, M. A., Glória, E. M. da, Salgado, J. M. \& Vizioli, M. R. (2002). Thermolysed and active yeast to reduce the toxicity of aflatoxin. Scientia Agricola, 59(2), 257-60.

Bochio, V., Takahashi, S. E., Groff, P. M., Schadeck, M. M. \& Maier, G. S. (2017). Efeitos da aflatoxina na produção avícola: Revisão. Revista PUBVET, 11(8), 832-39. 
Castro, I. M., Teixeira, A. S., Anjos, M. R. \& Santos, S. N. (2013). Contaminante em Leite: Análise de Aflatoxina M1 por Cromatografia Líquida de Alta Eficiência com Detecção por Fluorescência - CLAE/DFL. EMBRAPA, 2013.

Dias, A.S. (2018). Micotoxinas em produtos de origem animal. Revista Científica de Medicina Veterinária, 10(30), 1-15.

FAO/OMS. (2007). Codex Alimentarius: Producción de Alimentos de Origen Animal.

FDA (Food and Drug Administration). (2017). Bad Bug Book: foodborne pathogenic microorganisms and natural toxins handbooks aflatoxins 2017.Disponível em: https://www.fda.gov/food/bad-bug-book-second-edition/bbb-aflatoxin-structures.

Gonçalez, E., Felicio, J. D., Pinto, M. M., Rossi, M. H., Nogueira, J. H. C. \& Manginelli, S. (2005). Ocorrência de aflatoxina M1 em leite comercializado em alguns municípios do estado de São Paulo. Arq. Inst. Biol., São Paulo, 72(4), 435-8.

Gonçalves, L., Dalla, A. R., Gonzalez, S. L., Feltes, M. M. C., Badiale-Furlong, E. \& Dors, G. C. (2016). Determinação de aflatoxina m1 em leite bovino in natura. In: XXV Congresso Brasileiro de Ciência e Tecnologia de Alimentos: Alimentação: a árvore que sustenta a vida. FAURGS- Gramado, RS. 2016.

Gonçalves, B. L., Gonçalves, J. L., Rosim, R. E., Cappato, L. P., Cruz, A. G., Oliveira, C. A. F. \& Corassin, C. H. (2017). Effects of different sources of Saccharomyces cerevisiae biomass on milk production, composition and aflatoxin M1 excretion in milk from dairy cows fed aflatoxin B1. Journal of Dairy Science, 100 (7), 5701-08.

Gonçalves, B.L. (2018a). Uso de fontes de Saccharomyces cerevisiae na redução da excreção de aflatoxina M1 no leite de vacas leiteiras. Dissertação (Mestrado)- Faculdade de Zootecnia e Engenharia de Alimentos, Universidade de São Paulo. Pirassununga, 82f (a).

Gonçalves, K. D. M. (2018b). Ocorrência de aflatoxinas B1 e M1 em leite em pó e UAT, consumido em Cabo Verde e região sul do Brasil. Rio Grande, RS. 72f. Dissertação (Mestrado em Química Tecnológica e Ambiental)- Escola de Química e Alimentos. Universidade Federal do Rio Grande, Rio Grande do Sul.

Huang, L. C., Zheng, N., Zheng, B. Q., Wen, F., Cheng, J. B., Han R. W., Xu X. M., Li, S. L. \& Wang, J. Q. (2014). Simultaneous determination of aflatoxin M1, ochratoxin A, zearalenone and $\alpha$-zearalenol in milk, Food Chem, 1(146), 242-9.

Hussein, S.H. \& Brasell, J.M. (2001). Toxicity, metabolism, and impact of mycotoxins on humans and animals. Toxicology,167(1), 1-7.

IBGE - 2020. Estatística da Produção Pecuária. Disponível em: <https://ftp.ibge.gov.br/Producao_Pecuaria/Fasciculo_Indicadores_IBGE/abate-leite-couroovos_202004caderno.pdf>.

Iqbal, S. Z., Asic, M. R., Jinapa, S. \& Rashide, U. (2014). Detection of aflatoxins and zearalenone contamination in wheat derived products. Food Control, $35(1), 223-26$

Jager, A. V., Tedesco, M. P., Souto, P. C. M. C. \& Oliveira, C. A. F.(2013). Assessment of aflatoxin intake in São Paulo, Brazil. Food Control, 33(1), 87-92.

Lamiaa, M. S., Ahlam A. EL-L., Hussein, A. EL-M. (2019). Prevalence of Aflatoxins M1 and M2 in some curd dairy products. Alexandria Journal of Veterinary Sciences. AJVS. 61(1): 140-145.

Oliveira, C. A. F., Sebastião, L. S., Fagundes, H., Rosim, R. E. \& Fernandes, A. M. (2010). Determinação de aflatoxina B1 em rações e aflatoxina M1 no leite de propriedades do Estado de São Paulo. Ciência e Tecnologia de Alimentos, 30(1), 1-5.

Prado, G., Oliveira, M. S., Lima, A. S. \& Moreira, A. P. A. (2008). Ocorrência de aflatoxina M 1 em queijo parmesão consumido em Minas Gerais, Brasil. Ciênc. agrotec. 32.

Sabino, M., Purchio, A. \& Zorzeto, M. A. P. Variations in the levels of aflatoxin in cows milk consumed in the city of São Paulo. Food Additives and Contaminants, 6, 321-326, 1989.

Santos, J. S., França, V. R., Katto, S. \& Santana, E. H. W. (2015). Aflatoxina M1 em leite UHT pasteurizado e leite em pó comercializado em Londrina, Brasil e estimativa de exposição. ALAN vol.65 n 3. Conjunto de Caracas.

Saraiva, O. J. (2017). Determinação da aflatoxinas m1 em queijos coloniais comercializados na região vale do taquari -RS. Dissertação de Mestrado, UFRS 2017.

Sartori, A. V., Mattos, J. S., Moraes, M. H. P. \& Nóbrega, A. W. (2015). Determination of aflatoxins M1, M2, B1, B2, G1, G2 and ochratoxin A in UHT and powdered milk by modified quechers method and ultra-high performance liquid chromatography-tandem mass spectrometry. Food Analytical Methods, 8(1), $2321-30$

Shundo, L. \& Sabino, M.(2006). Aflatoxin M1 in milk by immunoaffinity column cleanup with TLC/HPLC determination. Brazilian Journal of Microbiology, São Paulo, 2006.

Tittlemier, S., Cramer, B., Dall asta, C., Iha, M., Lattanzio, V., Maragos, C., Solfrizzo, M., Stranska-Zachariasova, M., Stroka, J. \& Symarah, M. (2020) Developments in mycotoxin analysis: an update for 2018-19, World Mycotoxin Journal, 13 (1), 3-24.

Venâncio, R.L.(2017). Ocorrência e sazonalidade de aflatoxina m1 em leite nas regiões de Londrina-PR e Castro-PR. Dissertação (Mestrado em Ciência e Tecnologia de leite e derivados). 33f. UNOPAR.

Zheng, N., Sun, P., Wang, J. Q., Zhen, Y. P., Han, R. W. \& Xu, X. M. (2013). Occurrence of aflatoxin M1 in UHT milk and pasteurized milk in China Market. Food Control, 22(1),198-201. 